\title{
Developmental Changes in the Transmitter Properties of Sympathetic Neurons That Innervate the Periosteum
}

\author{
Stephen E. Asmus, Sarah Parsons, and Story C. Landis \\ National Institute of Neurological Disorders and Stroke, National Institutes of Health, Bethesda, Maryland 20892
}

\begin{abstract}
During the development of sweat gland innervation, interactions with the target tissue induce a change from noradrenergic to cholinergic and peptidergic properties. To determine whether the change in neurotransmitter properties that occurs in the sweat gland innervation occurs more generally in sympathetic neurons, we identified a new target of cholinergic sympathetic neurons in rat, the periosteum, which is the connective tissue covering of bone, and characterized the development of periosteal innervation of the sternum. During development, sympathetic axons grow from thoracic sympathetic ganglia along rib periosteum to reach the sternum. All sympathetic axons displayed catecholaminergic properties when they reached the sternum, but these properties subsequently disappeared. Many axons lacked detectable immunoreactivities for vesicular ace-
\end{abstract}

tylcholine transporter and vasoactive intestinal peptide when they reached the sternum and acquired them after arrival. To determine whether periosteum could direct changes in the neurotransmitter properties of sympathetic neurons that innervate it, we transplanted periosteum to the hairy skin, a noradrenergic sympathetic target. We found that the sympathetic innervation of the transplant underwent a noradrenergic to cholinergic and peptidergic change. These results suggest that periosteum, in addition to sweat glands, regulates the neurotransmitter properties of the sympathetic neurons that innervate it.

Key words: cholinergic differentiation factor; neural crest; sweat glands; synapse formation; neuropoietic cytokines; transmitter plasticity; autonomic neurons
Acquisition of the diverse array of neurotransmitter phenotypes displayed by distinct neuron populations is an important event in synapse development. Most neurons contain a small molecule neurotransmitter and one or more neuropeptides, and each neuron class expresses a characteristic complement of transmitter(s) and neuropeptides. Many neurons acquire the ability to produce a small molecule transmitter before their axons reach targets and form contacts. Ventral mesencephalic neurons contain tyrosine hydroxylase $(\mathrm{TH})$ before contacting striatum or limbic forebrain (Specht et al., 1981), dividing sympathetic precursors contain catecholamines (Cochard et al., 1979; Teitelman et al., 1979), and glutamic acid decarboxylase is present in growth cones (Mclaughlin et al., 1975). Similarly, neuropeptide expression can precede target contact. Sympathetic precursors contain neuropeptide Y, and its expression is correctly regulated in culture without target tissues (Tyrrell and Landis, 1994; Hall and MacPhedran, 1995). Furthermore, retinal ganglion cells express substance P independent of tectal interactions (Yamagata and Sanes, 1995). In some instances, target tissues influence neuropeptide expression in developing neurons (Coulombe and Nishi, 1991; Horgan and van der Kooy, 1992; Darland and Nishi, 1998). Target-derived differentiation factors, however, play a key role in determining both

\footnotetext{
Received Aug. 19, 1999; revised Nov. 24, 1999; accepted Dec. 3, 1999.

This work was supported by National Institutes of Health Grants NS023678 (S.C.L.) and NS09709 (S.E.A.), the Kentucky Academy of Sciences (S.E.A.), and the National Institute of Neurological Diseases and Stroke Intramural Research Program. We thank Dr. Robert J. Schotzinger, who played an important role in early aspects of the project, Dr. Ali Roghani, who kindly provided the vesicular acetylcholine transporter antiserum, Xiang-Hong Song for assistance with preliminary experiments, and Hua Tian for technical assistance.

Correspondence should be addressed to Dr. Story C. Landis at the above address. E-mail: slandis@codon.nih.gov.

Dr. Asmus's present address: Department of Biochemistry and Molecular Biology, Centre College, Danville, KY 40422.

Copyright (C) 2000 Society for Neuroscience $\quad 0270-6474 / 00 / 201495-10 \$ 15.00 / 0$
}

small molecule and neuropeptide transmitters in sympathetic neurons innervating sweat glands. Initially, these neurons display noradrenergic properties, whereas the mature innervation is cholinergic and contains vasoactive intestinal peptide (VIP) (Landis and Keefe, 1983; Leblanc and Landis, 1986; Landis et al., 1988). The conversion is governed by sweat gland-derived factor(s) (Schotzinger and Landis, 1988; Guidry and Landis, 1998b).

The target-mediated specification of both a small molecule transmitter and a neuropeptide in sweat gland neurons, although interesting, has not been demonstrated in any other neuronal population, including other sympathetic neurons. In vivo, cholinergic and VIP-containing neurons are present in sympathetic ganglia innervating sweat glands (Lundberg et al., 1979; Lindh, 1989; Morales et al., 1995). In addition, neurons in thoracic sympathetic ganglia, which do not innervate sweat glands, contain VIP (Lundberg et al., 1979; Kummer and Heym, 1988). Because VIP is coexpressed with choline acetyltransferase (ChAT) (Morales et al., 1995), we asked whether VIP-containing thoracic neurons are cholinergic. Periosteum, the connective tissue covering of bone, is a candidate target for cholinergic sympathetic innervation. Adult periosteum contains VIP-immunoreactive fibers associated with periosteum, as well as catecholaminergic fibers associated with blood vessels (Hohmann et al., 1986; Hill and Elde, 1991). Tracing studies indicate that periosteal VIP-IR fibers of the ribs and sternum originate from thoracic sympathetic ganglia (Hohmann et al., 1986).

Using an antiserum recognizing vesicular acetylcholine transporter (VAChT), we found that periosteum is a target of cholinergic sympathetic neurons. Analysis of developing innervation of the periosteum of sternum revealed that catecholaminergic properties were uniformly present, whereas cholinergic properties and VIP were often absent and appeared subsequently. Furthermore, VAChT and VIP were induced in fibers innervating periosteum 
transplanted under hairy skin. Together with studies of sweat gland innervation, these results suggest a common mechanism regulating cholinergic phenotype in several populations of sympathetic neurons and raise the possibility that target instruction plays a role in neurotransmitter determination more generally.

\section{MATERIALS AND METHODS}

Animals and surgery. Sprague Dawley rats (Zivic Miller, Zelienople, PA) were used for analyses of periosteal innervation in developing and adult rats, whereas inbred Lewis rats (Charles River Laboratories, North Wilmington, MA) were used in the transplantation experiments. No differences were observed in transmitter-related properties of periosteal innervation of Sprague Dawley and Lewis rats, and male and female rats of both strains were used.

Some neonatal rats were sympathectomized by intraperitoneal injections on postnatal day 1 (P1) to $\mathrm{P} 7 \mathrm{of} 100 \mathrm{mg} / \mathrm{kg}$ 6-hydroxydopamine hydrobromide (6-OHDA) (Sigma, St. Louis, MO) dissolved in physiological saline containing $1 \mathrm{mg} / \mathrm{ml}$ ascorbic acid. Littermate control animals received injections of vehicle. Treated and control animals were perfused on $\mathrm{P} 4$ or as adults. Unfixed tissue was also removed and frozen from rats at these same ages (P4: 6-OHDA-treated, $n=7$, vehicle, $n=$ 7; adults: 6-OHDA-treated, $n=7$, vehicle, $n=6$ ).

To transplant periosteum under hairy skin, the sternum was removed from P2 Lewis rats, and a rectangular segment of periosteal rudiment was lifted off the sternum with a scalpel and transplanted under the skin of P1 recipient animals. Recipients were anesthetized by hypothermia, and a semicircular flap was cut in the skin of the lateral thorax. The flap of skin was closed over the transplanted tissue and sealed with a pyroxylin solution (New-Skin; Medtech Labs, Cody, WY). After the New-Skin had dried, the pups were returned to their mother. Transplants were examined at $1(n=6), 2(n=6), 4(n=7)$, or $6(n=7)$ weeks after transplantation. Some pups receiving transplants also received injections of 6-OHDA as described above. These were analyzed $2(n=3)$ or $6(n=$ 2 ) weeks after surgery. To control for the effects of surgery, pieces of skin from the thorax of P2 rats were inserted under the skin flaps of two animals.

Histology. To examine the morphology of adult periosteum in situ and of mature transplants, rats were perfused transcardially with $2 \%$ paraformaldehyde and $2 \%$ glutaraldehyde in $0.1 \mathrm{M}$ phosphate buffer. Periosteum was dissected from the sternum, and periosteal transplants were dissected with the surrounding skin. Tissues were stored in the perfusion fixative until processing. After rinsing in phosphate buffer, tissues were post-fixed in $2 \%$ osmium tetroxide in $\mathrm{H}_{2} \mathrm{O}$ and stained en bloc with uranyl acetate, dehydrated, and embedded in Epon resin (Polysciences, Warrington, PA). One micrometer sections were stained with alcoholic toluidine blue.

Histochemistry and immunocytochemistry. The histochemical and immunocytochemical characteristics of the developing periosteal innervation of the sternum and the ventral portion of the adjacent ribs were examined at different ages. Embryos were removed from the uterus of anesthetized mothers on embryonic day 15 (E15), E16, E17, E18, or E20, and the sternum was either fresh frozen or fixed by immersion in paraformaldehyde. At the earlier ages, the entire rib cage was fixed and sectioned. After birth, tissue was removed from unfixed or perfusionfixed animals on P0, P4, P8, P14, or P21 and at 6-8 weeks. Tissue from at least five animals was examined at each stage, except at E20 and P8 when three animals were studied. Because the bone is not completely ossified until after P21, cryostat sections were cut through the entire sternum for all ages except the adult. The periosteum of adult animals was lifted from the bone before sectioning by removing rectangular flaps of periosteum from the dorsal (internal) surface of the sternum with a scalpel.

Endogenous catecholamines were localized in 12-15 $\mu \mathrm{m}$ cryostat sections of fresh frozen tissue using the glyoxylic acid-induced fluorescence technique of de la Torre (1980). Acetylcholinesterase (AChE) histochemistry was performed on both perfusion-fixed and fresh-frozen tissue sections with similar results. Fresh-frozen sections were immersed in $4 \%$ paraformaldehyde for $5 \mathrm{~min}$ before processing. All sections were washed in PBS and reacted as described previously (Landis and Keefe, 1983). The reaction mixture contained $1 \mathrm{~mm}$ tetra-isopropylpyrophosphoramide (Sigma), an inhibitor of nonspecific esterases.

For immunocytochemistry, embryonic tissue was immersed in $4 \%$ paraformaldehyde in $0.1 \mathrm{M}$ phosphate buffer, $\mathrm{pH} 7.4$, for 1-2 $\mathrm{hr}$ at room temperature. Postnatal rats were perfused transcardially with this fixative for $10 \mathrm{~min}$, after which the sternum was removed and post-fixed for an additional $50 \mathrm{~min}$. Adult rats were perfused with $50 \mathrm{ml}$ of PBS before perfusion with fixative. Fixed tissue was equilibrated in $30 \%$ sucrose in PBS containing $0.01 \%$ sodium azide, and frozen sections $(12 \mu \mathrm{m})$ were cut and mounted onto gelatin-coated slides. For TH, VIP, and calcitonin gene-related peptide (CGRP) immunolabeling, tissue sections were preincubated for $1 \mathrm{hr}$ in dilution buffer ( $0.01 \mathrm{~m}$ phosphate buffer, $\mathrm{pH} 7.4,0.5$ $\mathrm{M} \mathrm{NaCl}, 0.01 \%$ sodium azide, and 5\% bovine serum albumin), incubated overnight with primary antisera diluted in this buffer, rinsed in PBS, incubated with secondary antisera in dilution buffer containing $4 \%$ rat serum for $2 \mathrm{hr}$, rinsed, and then mounted with glycerol/PBS (1:1). All incubations were conducted at room temperature. For VAChT immunocytochemistry, sections were incubated with the primary antiserum for $3-5 \mathrm{~d}$ at $4^{\circ}$, followed by sequential, room temperature incubations with biotinylated goat anti-rabbit (1:200; Jackson ImmunoResearch, West Grove, PA) in dilution buffer with 5-10\% rat serum for $1 \mathrm{hr}$ and with Cy3-conjugated streptavidin (1:20,000; Jackson ImmunoResearch) in dilution buffer with no serum for $30 \mathrm{~min}$.

Sections were single- or double-labeled using primary antisera raised in different species and species-appropriate secondary antisera conjugated to distinct fluorochromes. Combinations of the following antisera were used: rabbit anti-VAChT [1:4000; gift of A. Roghani, Texas Tech University, Lubbock, TX; raised against a synthetic peptide from the C terminus;(Roghani et al., 1998)], guinea pig anti-VIP [1:300; generated in our laboratory and specific for VIP (Tyrrell and Landis, 1994)], rabbit anti-TH (1:200; Pel-Freez Biologicals, Rogers, AR), and rabbit antiCGRP (1:500; Amersham, Arlington Heights, IL). The secondary antisera used were fluorescein isothiocyanate-conjugated goat anti-rabbit (1:200; Cappel, Durham, NC) and rhodamine-conjugated goat antiguinea pig (1:200; Jackson ImmunoResearch). These secondary antisera bound only to the appropriate primary antiserum.

\section{RESULTS}

\section{Properties of adult periosteal innervation}

To determine whether the thoracic sympathetic neurons that contain VIP are cholinergic, sections of thoracic ganglia from adult rats were double-labeled with an antiserum against VIP and an antiserum that recognizes the cholinergic marker VAChT (Roghani et al., 1998). As expected, principal sympathetic neurons were encircled by punctate VAChT-IR cholinergic terminals arising from preganglionic fibers (Fig. 1b). In addition, every section contained several neuron cell bodies that possessed immunoreactivity for both VIP and VAChT (Fig. 1a,b).

After screening several candidate bones, we chose to examine the periosteum of the sternum. The sternum possessed a relatively flat surface and presented the largest area for analysis. In addition, its sympathetic innervation arises from thoracic ganglia (Hohmann et al., 1986), which contain cholinergic and VIPcontaining neurons but are not a source of sweat gland innervation. Adult rat periosteum consists of an outer fibrous layer sparsely populated by fibroblasts and an inner cellular layer containing osteoblasts and osteoblast progenitor cells (Fig. 1c). As described previously, (Hohmann et al., 1986; Bjurholm et al., 1988; Hill and Elde, 1991), VIP-IR fibers were observed to course through the periosteum (Fig. $2 a$ ).

To determine whether the periosteum receives cholinergic innervation, sections were single-labeled with the VAChT antiserum, double-labeled with both VAChT and VIP antisera, or reacted for AChE activity. Fibers immunoreactive for VAChT or reactive for $\mathrm{AChE}$ were identical in number and distribution to those containing VIP immunoreactivity (Fig. $2 a, c, d$ ). After double labeling for VAChT and VIP, all labeled axons contained both markers (Fig. $2 e, f$ ). The majority of the VAChT-VIP-IR fibers ran individually in the periosteum, but small bundles of axons were also observed. Fibers oriented parallel to the length of the bone were present in both the fibrous and cellular layers of the periosteum. When sections were double-labeled for VIP and TH, 

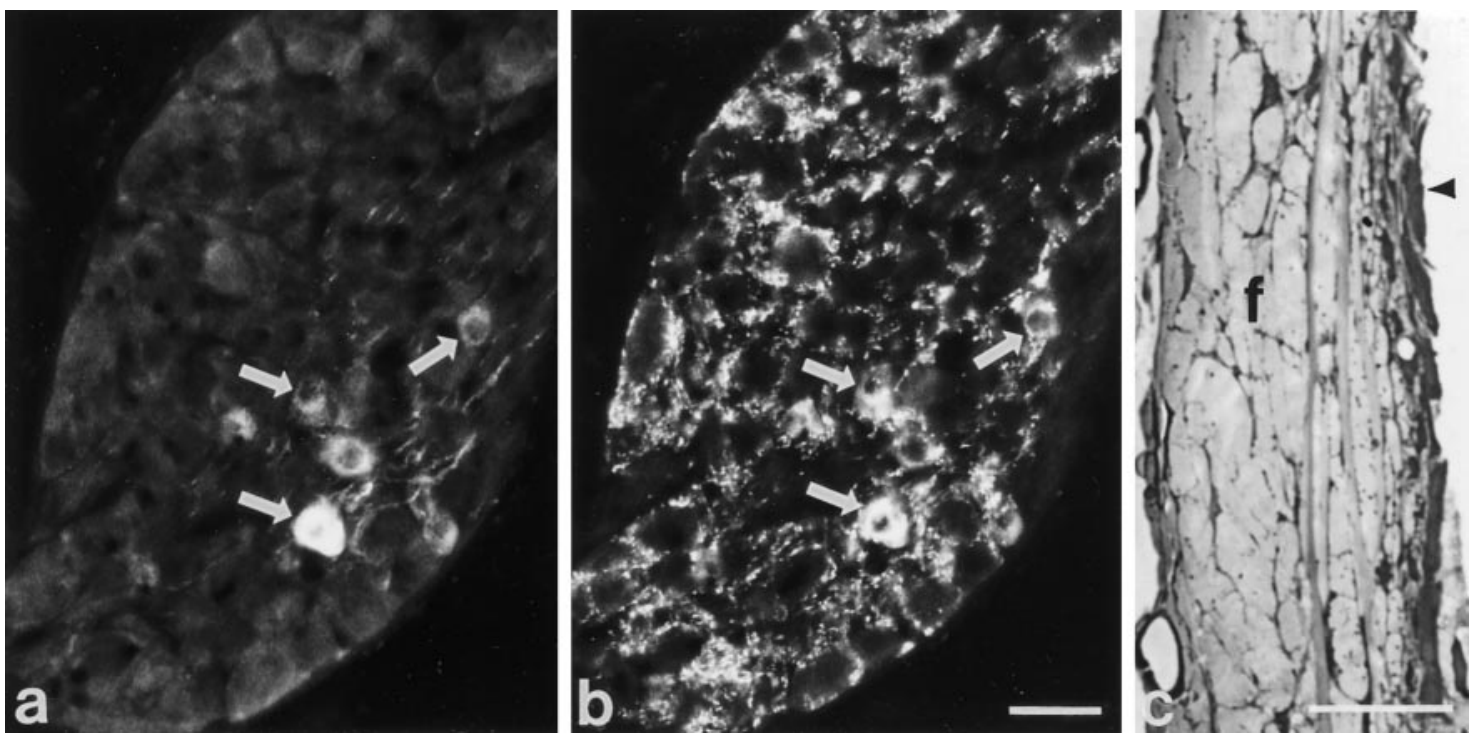

Figure 1. Adult rat thoracic sympathetic ganglion and periosteum. $a, b$, Photomicrographs of a single section through a thoracic sympathetic ganglion double-labeled for VIP with an antiserum raised in guinea pig and VAChT using an antiserum raised in rabbit. Several of the cell bodies present in this section that are immunoreactive for both VIP $(a)$ and VAChT $(b)$ are indicated by arrows. The dense plexus of preganglionic fibers that surround the neuron cell bodies also contain VAChT immunoreactivity $(b) . c$, A $1 \mu \mathrm{m}$ plastic section of periosteum stained with toluidine blue shows the inner cellular (arrowhead) and outer fibrous $(f)$ layers. Scale bars, $40 \mu \mathrm{m} . a$ and $b$ are the same magnification.

most VIP-IR fibers exhibited extremely faint immunoreactivity for TH (Fig. 2a,b), similar to that observed in the sweat gland innervation of mature rats (Landis et al., 1988). In contrast, axons associated with blood vessels in the periosteum and surrounding connective tissue and muscle displayed intense TH immunoreactivity and catecholamine histofluorescence but no VIP or VAChT immunoreactivity (Fig. $2 g, h$ ). Thus, the VIP-IR fibers, previously shown to be sympathetic in origin (Hohmann et al., 1986; Hill and Elde, 1991), are distinct from the sympathetic fibers innervating vasculature and are cholinergic rather than noradrenergic.

\section{Development of sympathetic periosteal innervation of the sternum}

To determine how and when sympathetic axons reach the sternum, which is located at the ventralmost extension of the ribs, we examined sections of the thoracic region of embryos from E15 to birth. Clearly defined ribs comprised of chondrocytes surrounded by perichondrium appeared in the lateral body wall at approximately E15. At this age, TH-IR sympathetic neurons were present in thoracic ganglia, but TH-IR axons were not observed in the thoracic region. Bundles of intensely stained VAChT-IR axons, presumably motor in origin, were observed adjacent to the ribs and in the presumptive intercostal muscles. TH-IR fibers were observed exiting the thoracic ganglia on E17 and growing toward the dorsalmost end of the ribs and along the ribs toward the sternum (Fig. 3). On E18, bundles of TH-IR fibers were observed in the lateral body wall, associated with the ribs and sternum. Thus, sympathetic fibers that innervate periosteum of the sternum travel ventrally along the rib periosteum to reach the sternum.

The initial sympathetic innervation of the periosteal covering of the sternum exhibited catecholaminergic characteristics, which diminished with age. Fibers displaying catecholamine histofluorescence and TH-IR were first observed in the periosteum of the sternum and adjacent ribs on E18, and their density increased between E18 and P0 (Fig. 4a,b). During development as in adulthood, periosteal fibers could be distinguished from those associated with blood vessels because the latter exhibited a characteristic circular or parallel arrangement. Catecholamine fluorescent fibers were observed in the periosteum through P8. At $\mathrm{P} 14$, the number of nonvascular catecholamine-containing fibers was significantly reduced, and at P21, only occasional, faintly fluorescent fibers were observed. In adult periosteum, nonvascular catecholamine fluorescent fibers were extremely rare, although intensely fluorescent fibers were observed surrounding blood vessels in and adjacent to the periosteum.

In contrast to the decreased expression of the catecholaminergic markers, the expression of cholinergic and peptidergic properties increased. At all ages examined, VAChT and VIP were colocalized in periosteal fibers (Fig. 4d,e), indicating that these markers were coexpressed and therefore labeled the same population of fibers. Sparse VAChT- and VIP-IR fibers were observed between $\mathrm{E} 18$ and $\mathrm{P} 0$ in the sternum and adjacent, ventral regions of the ribs. Their density increased dramatically after birth. During the first and second postnatal weeks, VAChT- and VIP-IR fibers attained the distribution and density seen in mature periosteum. When we examined the presence of catecholaminergic properties in the cholinergic-VIPergic fibers in the periosteum of the sternum by double labeling for TH and VIP, we found that, at E18 and P0, approximately half the nonvascular TH-IR periosteal fibers lacked VIP (Fig. 4b,c), whereas the others contained immunoreactivity for both $\mathrm{TH}$ and VIP. At P4 through P8, in contrast, nearly all periosteal fibers in the sternum and adjacent ribs were double-labeled for both $\mathrm{TH}$ and VIP (Fig. 4f, $g$ ). At P14, VIP-IR fibers with only faint TH-IR were consistently observed, as well as fibers that still contained both VIP and intense immunoreactivity for TH. At P21 as in the adult, the majority of VIP-IR periosteal fibers displayed only faint TH-IR (Fig. 2b,c). AChE activity was not detectable in periosteal fibers until P8 (Fig. 4h), although staining was seen at earlier ages in motor nerve fibers and end plates in intercostal 

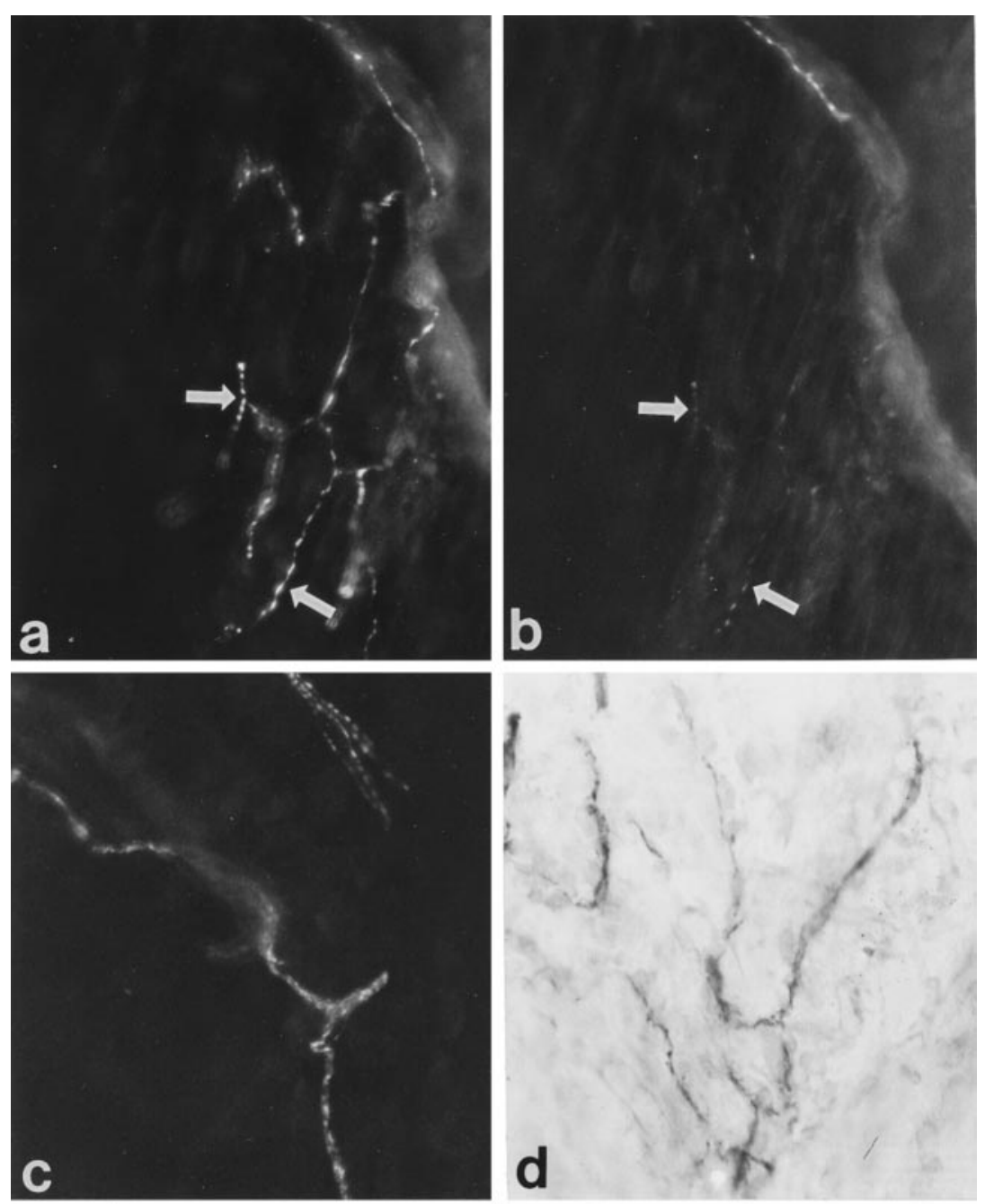

Figure 2. Transmitter-related properties of the sympathetic innervation of adult rat periosteum. $a, b$, Photomicrographs of a tangential section of periosteum doublelabeled for VIP and TH. Varicose axons coursing through the periosteum contain intense VIP $(a)$ and extremely faint TH $(b)$ immunoreactivity (arrows). At the top right in $a$, there is a single axon that is VIP-IR. This fiber is distinct from the intensely TH-IR fiber in $b$. $c, d$, Transmitter properties indicative of cholinergic neurons are also present in periosteal fibers. Fibers exhibiting punctate VAChT immunoreactivity $(c)$ and reactivity for $\operatorname{AChE}(d)$ are observed in the periosteum. $e, f$, To determine whether VIPergic and cholinergic properties are present in the same fibers, sections were double-labeled for VIP and VAChT. Periosteal fibers containing VIP immunoreactivity $(e)$ are also immunoreactive for VAChT $(f) . g$, Fibers associated with a blood vessel in the intercostal muscle adjacent to the sternum exhibit intense TH immunoreactivity compared with the faint TH immunoreactivity present in VIP-IR fibers $(b)$. VIP immunoreactivity is absent from TH-IR perivascular fibers $(h)$ (arrows). Scale bar: $h, 40 \mu \mathrm{m}$.
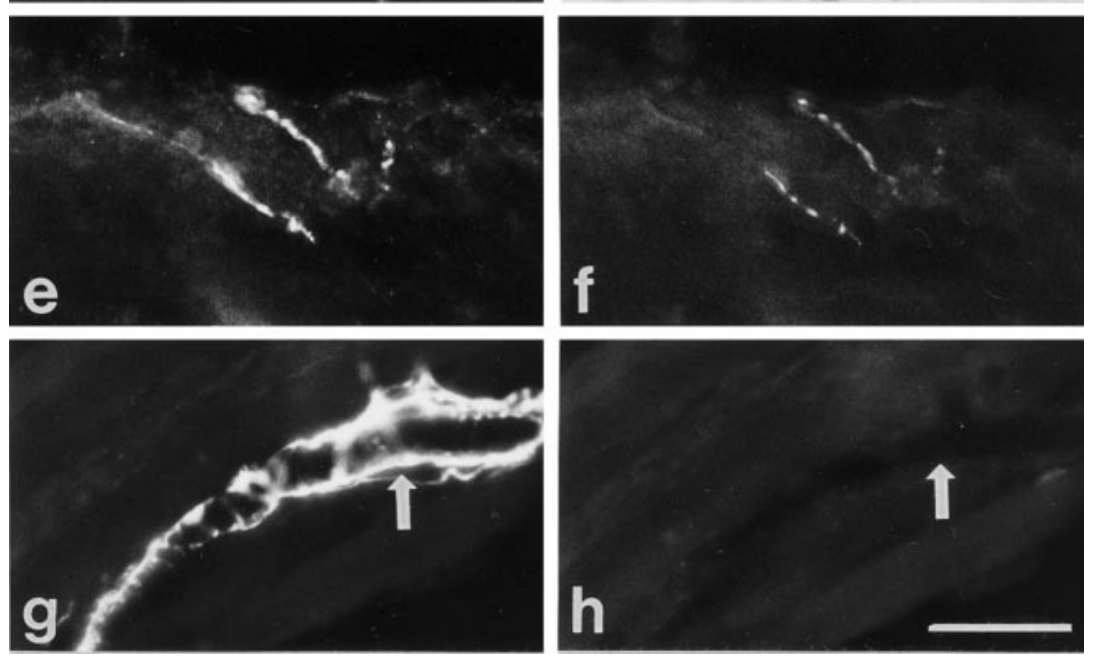

muscles. By P21, the density and distribution of periosteal AChEreactive fibers resembled those observed in adult periosteum.

\section{6-OHDA treatment eliminates cholinergic sympathetic innervation of periosteum}

To determine whether the cholinergic-VIPergic fibers in mature periosteum derived from the early catecholaminergic innervation, neonatal pups were treated with the neurotoxin 6-OHDA. When administered to neonatal rats, 6-OHDA, which is accumulated specifically by catecholaminergic neurons, destroys peripheral noradrenergic nerve terminals and cell bodies (Finch et al., 1973). We found that 6-OHDA treatment eliminated not only the fibers containing catecholamine fluorescence and TH-IR that were present in the periosteum of P4 pups but also the VAChT- and VIP-IR fibers (data not shown). Furthermore, staining for VAChT and VIP was virtually eliminated in the periosteum of adult rats that had been treated as neonates with 6-OHDA (Fig. 

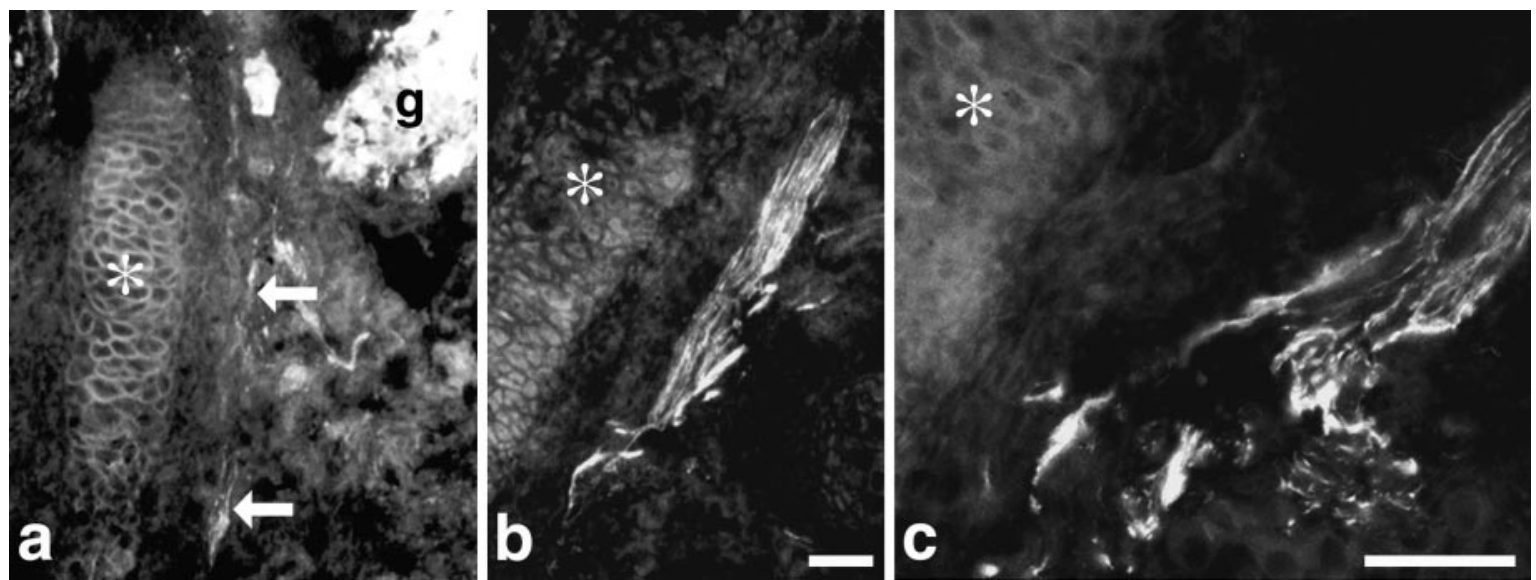

Figure 3. Development of periosteal innervation in the thoracic region. Axons from thoracic sympathetic neurons are closely associated with rib periosteum as they grow to the sternum. $a$, Bundles of TH-IR sympathetic axons were observed leaving the ganglion ( $g$ ) and extending along a rib (asterisk) on E17. The axons are indicated by arrows. b, TH-IR sympathetic axons (arrows) were observed in contact with the periosteum of the rib (asterisk) on E17. $c$, A bundle of TH-IR axons bifurcates, and some course along associated with the periosteum of the rib (asterisk). $a$ and $b$ are the same magnification. Scale bars: $a, b, 10 \mu \mathrm{m} ; c, 20 \mu \mathrm{m}$.

$5 b-d)$. In contrast, the CGRP-IR sensory innervation of the periosteum (Hill and Elde, 1991) was not affected (Fig. 5a). Together with the colocalization of TH and VIP in developing periosteal fibers, these results provide evidence that the cholinergic-VIPergic fibers in adult periosteum were initially catecholaminergic and that the sympathetic periosteal innervation underwent a transition in phenotype during development.

\section{Transplanted periosteum induces a transmitter switch}

When periosteum is transplanted heterotopically, it forms bone (Burman and Umansky, 1930). To determine whether the changes in transmitter properties described above could be the consequence of interactions with the periosteum, periosteal rudiments from $\mathrm{P} 2$ rats were transplanted under the skin of the lateral thorax of $\mathrm{P} 1$ rats. As expected, the transplanted periosteal rudiments formed small bones, or ossicles, in the subcutaneous connective tissue. The ossicles were $\sim 3-6 \mathrm{~mm}$ in length, consisted of cartilage and bone, and were surrounded by periosteum that was morphologically indistinguishable from normal periosteum (Fig. $6 a, b)$. The skin of the lateral thorax above the transplants developed into hairy skin containing noradrenergic sympathetic fibers innervating blood vessels and piloerectors and sensory fibers immunoreactive for CGRP (Schotzinger and Landis, 1990).

The transmitter properties of fibers innervating the periosteum of the transplants underwent a phenotypic transition resembling that displayed by the innervation of the periosteum developing in situ. One week after transplantation, only rare catecholaminepositive and TH-IR fibers, but no VIP-IR fibers, were observed. After 2 weeks, the number of nonvascular catecholamine fluorescent fibers associated with the periosteum had increased (Fig. $6 c$ ), and fibers immunoreactive for TH alone (Fig. 6d,e) or doublelabeled for TH and VIP were observed. In the periosteum of 4 week transplants, catecholamine-containing fibers were observed less frequently than at 2 weeks, and the number of VAChT- and VIP-IR fibers had increased (Fig. $6 g$ ). Double labeling for VIP and $\mathrm{TH}$ revealed that the VIP-IR fibers were either singlelabeled for VIP or exhibited only faint $\mathrm{TH}$ immunoreactivity (Fig. $6 g, h$ ). Six weeks after surgery, few catecholamine fluorescent or single-labeled TH-IR fibers were evident (Fig. $6 f$ ). As at 4 weeks, however, VAChT- and VIP-IR fibers were present, and the VIP-IR fibers contained either faint or no detectable $\mathrm{TH}$ immunoreactivity. In contrast to normal periosteal innervation, no AChE-reactive fibers were seen associated with the transplant or in the hairy skin, although the other transmitter-related properties characteristic of periosteal innervation appeared.

To confirm the sympathetic origin of the cholinergic and VIP-IR periosteal fibers, some pups receiving transplants were treated with 6-OHDA. This treatment resulted in the absence of catecholaminergic fibers in the periosteum and surrounding hairy skin 2 weeks after transplantation and dramatically reduced the number of VIP-IR, but not CGRP-IR, fibers in the periosteum of 6 week transplants (data not shown). To confirm that the transplantation procedure itself did not affect transmitter properties, a piece of skin was transplanted subcutaneously to the lateral thorax. Six weeks after transplantation, sympathetic and sensory innervation in the region of transplanted skin was identical to that described previously in adult rat hairy skin (Schotzinger and Landis, 1990), with no VAChT- or VIP-IR fibers present.

\section{DISCUSSION}

Periosteum is a previously unrecognized target of cholinergic sympathetic neurons. The similarities in the transmitter properties of the mature sympathetic innervation of periosteum and sweat glands are striking. Both display cholinergic properties, AChE reactivity, and VIP immunoreactivity (Sjoqvist, 1963; Lundberg et al., 1979; Landis and Keefe, 1983; Hohmann et al., 1986; Leblanc and Landis, 1986; Landis et al., 1988; Guidry and Landis, 1998b). In sweat glands, cholinergic function is evident from the findings that nerve-evoked secretion is muscarinic in pharmacology (Langley, 1891; Stevens and Landis, 1987) and sympathetic fibers innervating sweat glands release acetylcholine (Dale and Feldberg, 1934) and contain ChAT activity and ChAT immunoreactivity (Leblanc and Landis, 1986). The tools that were successful in revealing cholinergic function in sweat gland innervation are less readily applicable to periosteum. Although the presence of nicotinic acetylcholine receptor mRNA in avian periosteum (Romano et al., 1997) suggests that cholinergic sympathetic innervation influences periosteal function, in contrast to sweat glands, it is unclear what aspects of periosteal function are regulated by the cholinergic innervation. Assays of ChAT activity in periosteum are confounded by contaminating skeletal muscle 
Figure 4. Development of transmitter-related properties in the periosteum. $a-c$, Catecholaminergic properties are present in developing periosteal fibers. $a$, On E18, catecholamine histofluorescent fibers are seen in the periosteum (arrowheads), which runs vertically in the photomicrograph, adjacent to the cartilaginous sternum $(s)$. Catecholamine fluorescent fibers are also present surrounding a blood vessel that is adjacent to the sternum at the top of the photomicrograph. $b, c$, A single section through the sternum on P0 double-labeled for TH and VIP, showing a TH-IR periosteal fiber (b) that is not immunoreactive for $\operatorname{VIP}(c) . d, e$, Between E18 and P0, approximately half of the TH-IR fibers also contain VIP immunoreactivity, and these VIP-IR fibers, shown here in P0 periosteum $(d)$, are also immunoreactive for VAChT (e). $s$, Cartilage of sternum in $a-e . f-h$, One to 2 weeks after birth, a dual phenotype is evident in the majority of periosteal fibers, demonstrated in these photomicrographs of longitudinal sections through the periosteum of the sternum. $f, g$, On P14, intense VIP $(f)$ and TH $(g)$ immunoreactivities are colocalized in most periosteal fibers (arrows). $h$, AChE-reactive fibers are first detected in the periosteum on P8. Scale bar: $h, 40 \mu \mathrm{m}$.
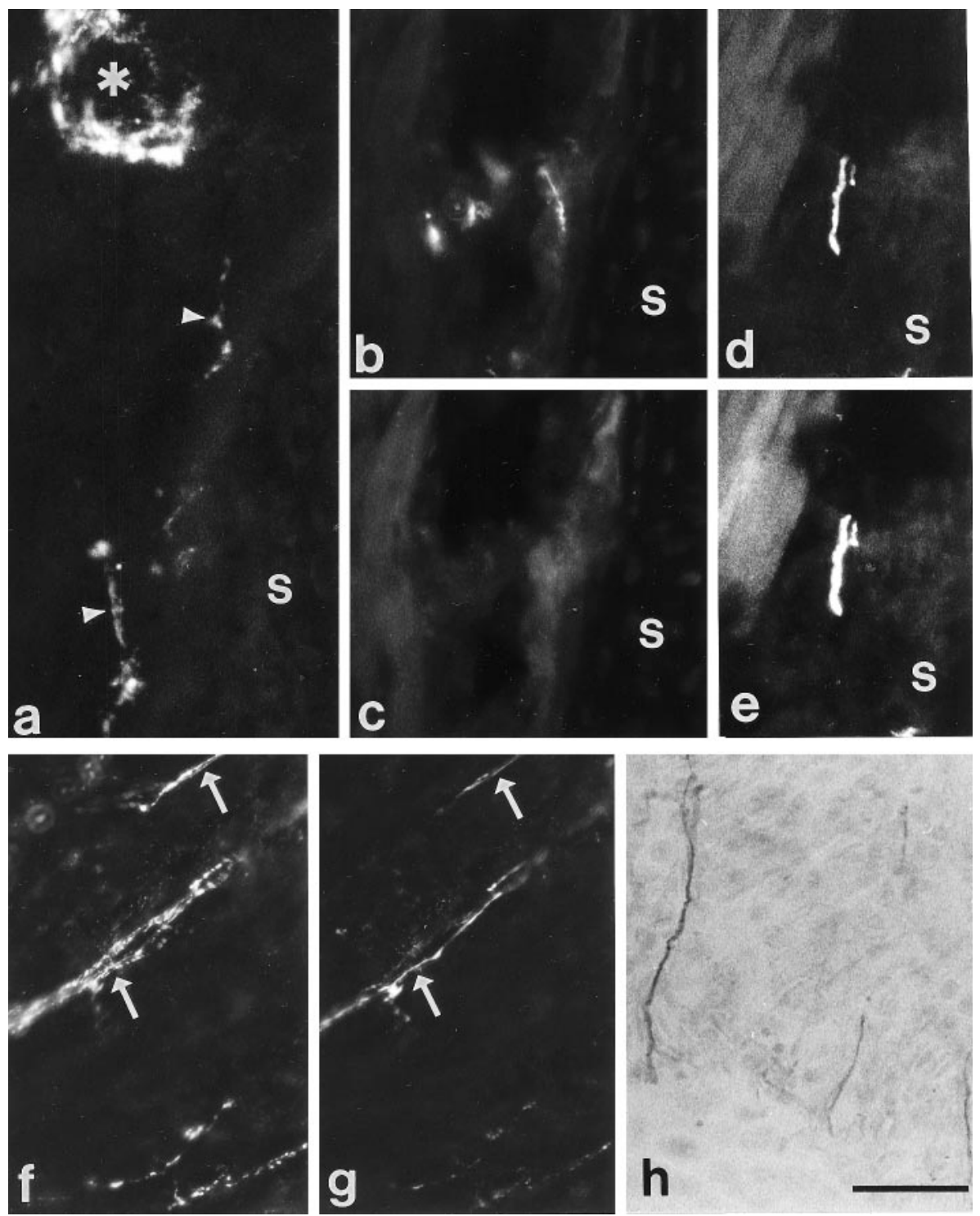

that remains attached after dissection. Finally, the ChAT antibodies presently available fail to label cholinergic sympathetic fibers in periosteum or sweat glands. The development of antisera recognizing VAChT, which transports acetylcholine into synaptic vesicles and is required for cholinergic transmission, has provided a new tool for mapping cholinergic terminals (Roghani et al., 1998; Schafer et al., 1998). The use of VAChT as a cholinergic marker is validated by the discovery that the gene encoding VAChT is embedded in and coordinately regulated with the gene encoding ChAT (Alfonso et al., 1994; Bejanin et al., 1994; Erickson et al., 1994; Berrard et al., 1995; Berse and Blusztajn, 1995; Misawa et al., 1995) and VAChT immunoreactivity coincides with ChAT immunoreactivity in cell bodies and terminal fields of classically defined cholinergic neurons (Roghani et al., 1998; Schafer et al., 1998).

Two lines of evidence indicate that the mature cholinergicVIPergic periosteal innervation initially displays catecholaminergic properties. First, immunoreactivities for VIP and TH and for VIP and VAChT were colocalized in developing periosteal axons. Although catecholamine fluorescence was absent from the mature innervation, indicating the absence of stored cat- echolamines, faint TH immunoreactivity, similar to that seen in mature sweat gland innervation (Landis et al., 1988), was detectable in many VIP-IR periosteal fibers. Second, when we treated neonatal rats with 6-OHDA, a specific noradrenergic neurotoxin, to eliminate developing catecholaminergic neurons (Finch et al., 1973), we found that VAChT-IR, VIP-IR, and AChE-reactive fibers were absent from $\mathrm{P} 4$ and adult periosteum. Furthermore, ChAT activity was reduced by half in homogenates of periosteum but not intercostal muscle (data not shown). Because VAChT immunoreactivity was absent from the periosteum of 6-OHDAtreated animals, the residual ChAT activity in homogenates is very likely to come from motor end plates in intercostal muscles, which adhere tightly to the periosteum and are difficult to remove completely during dissection. The developing sympathetic innervation of sweat glands is similarly sensitive to 6-OHDA (Yodlowski et al., 1984).

The results of the transplantation studies indicate that periosteum can induce alterations in the transmitter properties of sympathetic neurons that innervate it. When periosteal rudiments were implanted under the hairy skin of neonatal rats, sympathetic fibers that normally innervate noradrenergic targets in hairy skin, 

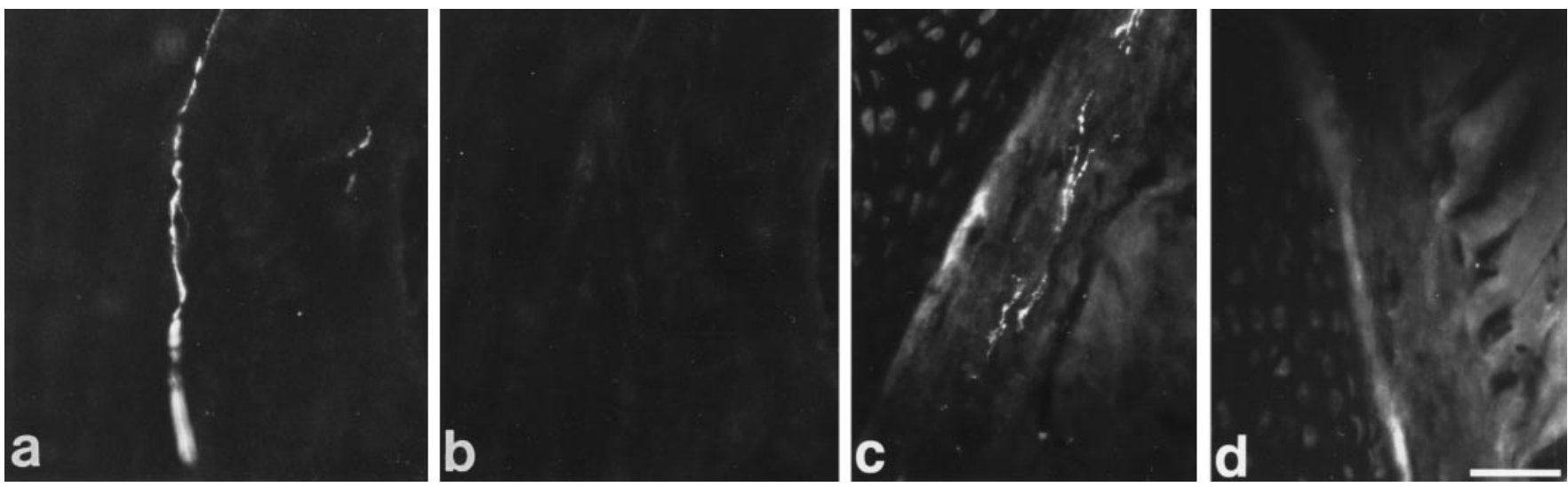

Figure 5. Neonatal 6-OHDA administration reduces the number of fibers that display cholinergic and VIPergic properties in mature periosteal fibers. $a, b$, Double labeling for CGRP and VIP in a section of periosteum from an adult rat treated neonatally with 6-OHDA demonstrates that CGRP-IR fibers, presumably of sensory origin, are not affected by this toxin $(a)$, but the number of VIP-IR fibers is reduced dramatically $(b)$. $c$, 6-OHDA treatment also reduces the number of VAChT-IR reactive fibers in the periosteum $(d)$ compared with control $(c)$. Scale bar: $a-d$, $20 \mu \mathrm{m}$.

piloerectors, and blood vessels (Schotzinger and Landis, 1990) became associated instead with the periosteum covering the ossicles that formed beneath the hairy skin. The axons innervating the transplanted tissue initially displayed catecholaminergic properties but subsequently acquired VAChT- and VIP-IR. This transition in phenotype, occurring in neurons that normally remain noradrenergic, is similar to that of developing periosteal innervation in situ. Our results are consistent with and extend those of Bjurholm and colleagues (1990), who observed VIP-IR fibers innervating the periosteum surrounding heterotopic bone induced by transplantation of bone matrix to abdominal muscles of adult rats. In these studies, however, the origin of the VIPimmunoreactive fibers was not determined. Furthermore, transplantation of bone matrix into adult muscle could have axotomized preexisting sympathetic axons and induced VIP expression (Hyatt-Sachs et al., 1993). In the present study, the VAChT- and VIP-immunoreactive fibers innervating the transplant were identified as sympathetic via colocalization of VIP and TH, as well as 6-OHDA sensitivity, and transplantation was performed at P1 before sympathetic or sensory fibers arrive in lateral thoracic skin (Schotzinger and Landis, 1990). Together, the results of our experiments provide evidence that periosteum, like sweat glands, can induce a cholinergic-VIPergic phenotype in sympathetic neurons. In contrast to the appearance of VAChT and VIP immunoreactivity in fibers innervating the transplanted periosteum, AChE reactivity, normally present in periosteal innervation after P7, was not detected. It is of interest in this regard that $\mathrm{AChE}$ reactivity is present in some, but not all, sympathetic fibers that innervate sweat glands transplanted to hairy skin, although catecholamines are uniformly lost and ChAT activity is acquired (Schotzinger et al., 1994). Acquisition of AChE may be regulated independently from other transmitter properties or alternatively hairy skin may contain an activity that inhibits the development of AChE.

Although the transplantation studies provide evidence that periosteum can induce cholinergic and peptidergic properties and reduce the expression of noradrenergic properties in sympathetic neurons in vivo, they do not establish that interactions with the periosteum are responsible for changes in normal periosteal innervation. When we examined the transmitter properties of sympathetic axons in periosteum of the sternum, we found that, although all fibers expressed $\mathrm{TH}$ immunoreactivity upon their arrival at the sternum between E18 and P0, half of them also exhibited VAChT and VIP immunoreactivity. This observation raised the possibility that some sympathetic neurons expressed cholinergic and peptidergic properties in addition to noradrenergic before interacting with the periosteum. Examination of younger embryos, however, revealed that after exiting the thoracic ganglia sympathetic fibers traveled in association with rib periosteum to reach the sternum and therefore would have been exposed to periosteal-derived cholinergic inducing factor(s) as they grew. Thus, although the periosteum of the sternum offers a number of experimental advantages for the developmental analysis of transmitter properties of periosteal innervation, it has the disadvantage of being the most ventral periosteum in the thorax and at the most distal reach of the sympathetic axons. The finding that the sweat gland-targeted innervation in Tabby mutant mice, which lack sweat glands, does not acquire VAChT or VIP immunoreactivities but maintains noradrenergic properties has provided compelling evidence for the essential role of the sweat glands in inducing changes in the sweat gland innervation (Guidry and Landis, 1998a). Because the periosteum is essential for bone formation during development, it is not possible at present to eliminate it and examine the effects of this manipulation on the transmitter properties of periosteal neurons.

Based on the induction of a similar transmitter repertoire, the periosteal factor and sweat gland factors are likely to be related or identical. The sweat gland factor appears to be a member of the neuropoietic cytokine family (Rao and Landis, 1990; Bazan, 1991; Patterson, 1992; Rao et al., 1992). Several members of this family, which includes ciliary neurotrophic factor (CNTF), leukemia inhibitory factor (LIF), and cardiotrophin-1 (CT-1), induce cholinergic and reduce noradrenergic properties in cultured sympathetic neurons (Fukada, 1985; Saadat et al., 1989; Yamamori et al., 1989; Pennica et al., 1995). Analyses of transgenic mice and culture studies provide no evidence, however, that the sweat gland factor is LIF, CNTF, or CT-1 (Rao et al., 1992, 1993; Habecker et al., 1995, 1997; Francis et al., 1997). Although the characterization of the periosteal factor is less well advanced than that of the sweat gland factor, studies to date suggest that it also does not correspond to either LIF or CNTF (Francis et al., 1997).

The effects of the change in transmitter properties on neural regulation of periosteal function are incompletely understood. 

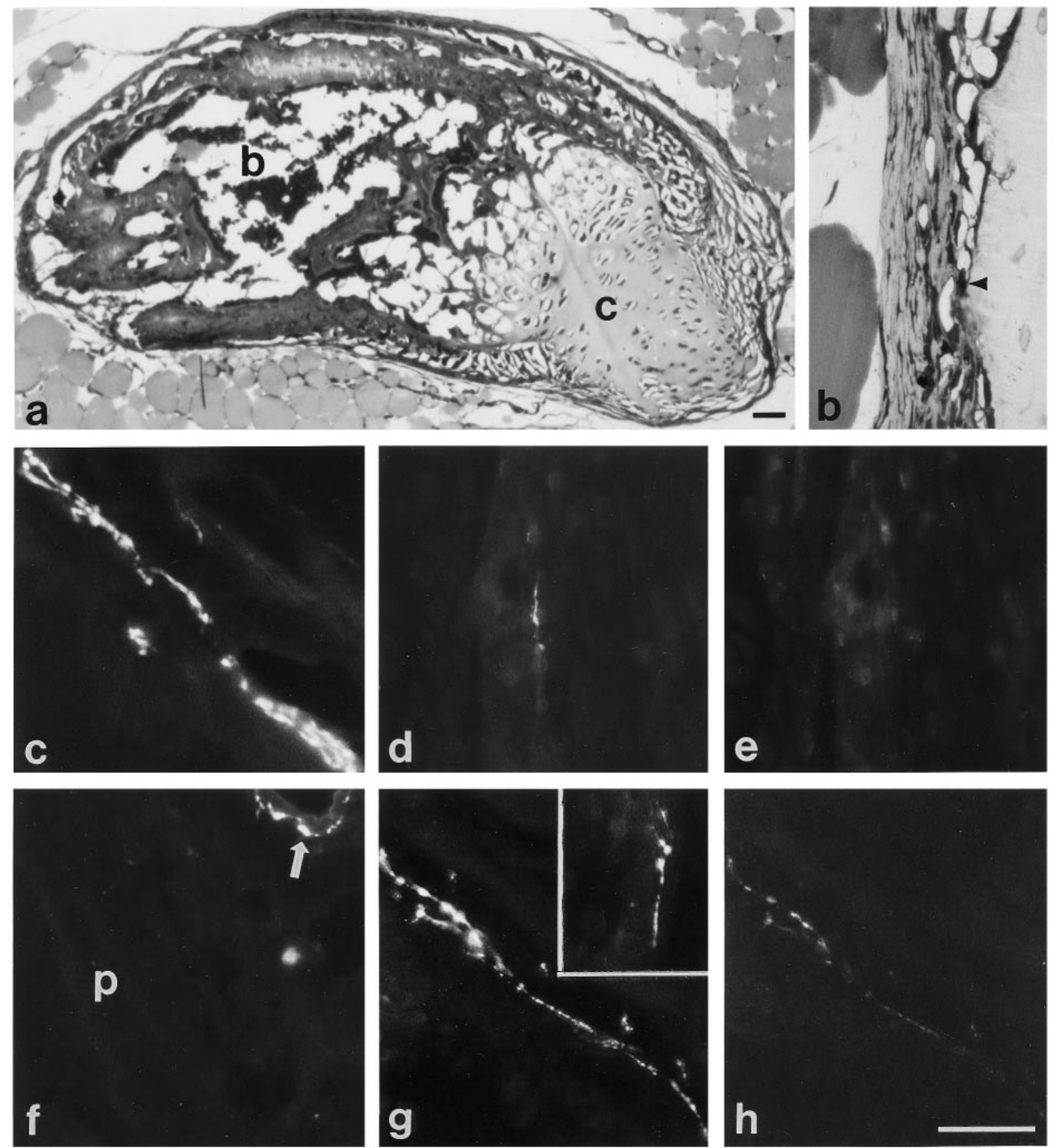

Figure 6. Development of transmitter properties of sympathetic fibers innervating ectopic periosteum. $a, b$, One micrometer plastic sections of 2 week transplants. $a$, Periosteal rudiments transplanted under the skin of the lateral thorax form a small bone, consisting of both cartilage $(c)$ and trabecular bone with intervening marrow spaces $(b) . b$, This photomicrograph shows a higher magnification view of the periosteum surrounding the transplants, which is similar in appearance to periosteum in situ, with an inner cellular layer (arrowhead) adjacent to the bone of the transplant. $c-e$, Sections of periosteal transplants 1-2 weeks after surgery. $c$, Sympathetic fibers that innervate the periosteum of the transplant initially contain catecholamine fluorescence. $d, e$, After double labeling for TH and VIP, periosteal fibers that contain TH $(d)$ but not VIP $(e)$ immunoreactivity are seen. $f-h$, Sections of periosteal transplants 4-6 weeks after surgery. $f$, Few catecholamine fluorescent fibers are observed in the periosteum $(p)$ of the transplant 6 weeks after transplantation, although blood vessels adjacent to the transplant are surrounded by catecholaminergic fibers (arrow). $g, h$, A section of a 4 week transplant double-labeled for VIP and TH contains intense VIP-IR fibers $(g)$, and these fibers exhibit only faint TH immunoreactivity $(h)$. $g$, Inset, VAChT-IR fibers are also observed in the periosteum of a transplant 4 weeks after surgery. Scale bars: $a, h, 40 \mu \mathrm{m} . b-h$ are the same magnification.

Because of its osteogenic potential, periosteum is crucial for bone development and fracture healing (Hall, 1970), and sympathetic input has been postulated to play a critical role in these processes. For example, neonatal chemical sympathectomy and adult surgical sympathectomy decrease osteoblast metabolism and increase bone remodeling or osteoclast activity (Singh et al., 1982; Sandhu et al., 1987, 1990; Hill et al., 1991). Evidence exists for a possible role of VIP in that cultured osteosarcoma cells possess VIP receptors (Hohmann and Tashjian, 1984) and VIP stimulates bone resorption in mouse calvaria in organ culture (Hohmann et al., 1983). Although embryonic chick periosteal cells express nicotinic receptor mRNA (Romano et al., 1997), no periosteal 
response to acetylcholine has been described. The lack of understanding of the functional consequences of the target-induced change in transmitter properties in periosteal innervation is in marked contrast to sweat glands in which the induction of cholinergic function in the innervation is required to trigger and maintain secretory responsiveness (Stevens and Landis, 1987; Grant et al., 1995).

\section{REFERENCES}

Alfonso A, Grundahl K, McManus JR, Asbury JM, Rand JB (1994) Alternative splicing leads to two cholinergic proteins in Caenorhabditis elegans. J Mol Biol 241:627-630.

Bazan JF (1991) Neuropoietic cytokines in the hematopoetic fold. Neuron 7:197-208.

Bejanin S, Cervini R, Mallet J, Berrard S (1994) A unique gene organization for two cholinergic markers, choline acetyltransferase and a putative vesicular transporter of acetylcholine. J Biol Chem 269:21944-21947.

Berrard S, Varoqui H, Cervini R, Israel M, Mallet J, Diebler M-F (1995) Coregulation of two embedded gene products, choline acetyltransferase and the vesicular acetylcholine transporter. J Neurochem 65:939-942.

Berse B, Blusztajn JK (1995) Coordinated up-regulation of choline acetyltransferase and vesicular acetylcholine transporter gene expression by the retinoic acid receptor alpha, cAMP, and leukemia inhibitory factor/ciliary neurotrophic factor signaling pathways in a murine septal cell line. J Biol Chem 270:22101-22104.

Bjurholm A, Kreicbergs A, Terenius L, Goldstein M, Schultzberg M (1988) Neuropeptide Y, tyrosine hydroxylase- and vasoactive intestinal polypeptide-immunoreactive nerves in bone and surrounding tissues. J Auton Nerv Syst 25:119-125.

Bjurholm A, Kreicbergs A, Dahlberg L, Schultzberg M (1990) The occurrence of neuropeptides at different stages of DBM-induced heterotopic bone formation. Bone Miner 10:95-107.

Burman MS, Umansky M (1930) An experimental study of free periosteal transplants wrapped around tendon. J Bone Joint Surg Am 12:579-594.

Cochard PM, Goldstein M, Black IB (1979) Initial development of the noradrenergic phenotype in autonomic neuroblasts of the rat embryo in vivo. Dev Biol 71:100-114.

Coulombe JN, Nishi R (1991) Stimulation of somatostatin expression in developing ciliary ganglion neurons by cells of the choroid layer. J Neurosci 11:553-562.

Dale HH, Feldberg W (1934) The chemical transmission of secretory impulses to the sweat glands of the cat. J Physiol (Lond) 82:121-123.

Darland DC, Nishi R (1998) Activin A and follistatin influence expression of somatostatin in the ciliary ganglion in vivo. Dev Biol 202:292-303.

de la Torre JC (1980) An improved approach to histofluorescence using the SPG method for tissue monoamines. J Neurosci Methods 3:1-5.

Erickson JD, Varoqui H, Schafer MK-H, Modi W, Diebler M-F, Weihe E, Rand J, Eiden LE, Bonner TI, Usdin TB (1994) Functional identification of a vesicular acetylcholine transporter and its expression from a "cholinergic" gene locus. J Biol Chem 269:21929-21932.

Finch L, Haeusler G, Thoenen H (1973) A comparison of the effects of chemical sympathectomy by 6-hydroxydopamine in newborn and adult rats. Br J Pharmacol 47:249-260.

Francis NJ, Asmus SE, Landis SC (1997) CNTF and LIF are not required for the target-directed acquisition of cholinergic and peptidergic properties by sympathetic neurons in vivo. Dev Biol 182:76-87.

Fukada K (1985) Purification and partial characterization of a cholinergic differentiation factor. Proc Natl Acad Sci USA 82:8795-8799.

Grant MP, Francis NJ, Landis S (1995) The role of acetylcholine in regulating secretory responsiveness in rat sweat glands. Mol Cell Neurosci 6:32-42.

Guidry G, Landis S (1998a) Developmental regulation of neurotransmitters in sympathetic neurons. Adv Pharmacol 42:895-898.

Guidry G, Landis SC (1998b) Target-dependent development of the vesicular acetylcholine transporter in rodent sweat gland innervation. Dev Biol 199:175-184.

Habecker BA, Pennica D, Landis SC (1995) Cardiotrophin-1 is not the sweat gland derived factor. NeuroReport 7:41-44.

Habecker BA, Symes AJ, Stahl N, Francis NJ, Economides A, Fink J,
Yancopoulos GD, Landis S (1997) A sweat gland-derived differentiation activity acts through known cytokine signaling pathways. J Biol Chem 272:30421-30428.

Hall AK, MacPhedran SE (1995) Multiple mechanisms regulate sympathetic neuronal phenotype. Development 121:2361-2371.

Hall BK (1970) Cellular differentiation in skeletal tissues. Biol Rev 45:455-484.

Hill EL, Elde R (1991) Distribution of cGRP-, VIP-, SP-, and NPYimmunoreactive nerves in the periosteum of the rat. Cell Tissue Res 264:469-480.

Hill EL, Turner R, Elde R (1991) Effects of neonatal sympathectomy and capsaicin treatment on bone remodeling in rats. Neuroscience 44:747-755.

Hohmann EL, Tashjian AH (1984) Functional receptors for vasoactive intestinal peptide on human osteosarcoma cells. Endocrinology 114:1321-1327.

Hohmann EL, Levine L, Tashjian AH (1983) Vasoactive intestinal peptide stimulates bone resporption via a cyclic adenosine 3,5'monophosphate-dependent mechanism. Endocrinology 112:1233-1239.

Hohmann EL, Elde RP, Rysavy JA, Einzig S, Gebhard RL (1986) Innervation of periosteum and bone by sympathetic vasoactive intestinal peptide-containing nerve fibers. Science 232:868-871.

Horgan K, van der Kooy D (1992) Visceral targets specify calcitonin gene-related peptide and substance $\mathrm{P}$ enrichment in trigeminal afferent projections. J Neurosci 12:1135-1143.

Hyatt-Sachs H, Schreiber R, Bennett T, Zigmond R (1993) Phenotypic plasticity in adult sympathetic ganglia in vivo: effects of deafferentation and axotomy on the expression of vasoactive intestinal peptide. J Neurosci 13:1642-1653.

Kummer W, Heym C (1988) Neuropeptide distribution in the cervicothoracic paravertebral ganglia of the cat with particular reference to calcitonin gene-related peptide immunoreactivity. Cell Tissue Res 252:463-471.

Landis SC, Keefe D (1983) Evidence for neurotransmitter plasticity in vivo: developmental changes in the properties of cholinergic sympathetic neurons. Dev Biol 98:349-372.

Landis SC, Schwab M, Siegel RE (1988) Evidence for neurotransmitter plasticity in vivo. II. Immunocytochemical studies of rat sweat gland innervation during development. Dev Biol 126:129-138.

Langley JN (1891) On the course and connections of the secretory fibers supplying the sweat glands of the feet of the cat. J Physiol (Lond) 12:347-369.

Leblanc G, Landis SC (1986) Development of choline acetyltransferase activity in the cholinergic sympathetic innervation of sweat glands. J Neurosci 6:260-265.

Lindh B, Lundberg J, Hokfelt T (1989) NPY-, galanin-, VIP/PHI-, CGRP- and substance P-immunoreactive neuronal subpopulations in cat autonomic and sensory ganglia and their projections. Cell Tissue Res 256:259-273.

Lundberg JM, Hokfelt T, Schultzberg M, Uvnas-Wallensten K, Kohler C, Said SI (1979) Occurrence of vasoactive intestinal polypeptide(VIP)like immunoreactivity in certain cholinergic neurons of the cat: evidence from combined immunohistochemistry and acetylcholinesterase staining. Neuroscience 4:1539-1559.

Mclaughlin BJ, Wood JG, Saito K, Roberts E, Wu JY (1975) The fine structural localization of glutamate decarboxylase in developing axonal processes and presynaptic terminals of rodent cerebellum. Brain Res 85:355-371.

Misawa H, Takahashi R, Deguchi T (1995) Coordinate expression of vesicular acetylcholine transporter and choline acetyltransferase in sympathetic superior cervical neurones. NeuroReport 6:965-968.

Morales MA, Holmberg K, Xu Z, Cozzari C, Hartman B, Emson P, Goldstein M, Elfvin L, Hokfelt T (1995) Localization of choline acetyltransferase in rat peripheral sympathetic neurons and its coexistence with nitric oxide synthase and neuropeptides. Proc Natl Acad Sci USA 92:11819-11823.

Patterson P (1992) The emerging neuropoietic cytokine family: first CDF/LIF, CNTF and IL-6; next ONC, MGF GCSF? Curr Opin Neurobiol 2:94-97.

Pennica D, Shaw KJ, Swanson TA, Moore MW, Shelton DL, Zioncheck KA, Rosenthal A, Taga T, Paoni NF, Wood WW (1995) Cardiotrophin-1: biological activities and binding to the leukemia inhibitory factor receptor/gp130 signalling complex. J Biol Chem 270:10915-10922. 
Rao M, Landis S (1990) Characterization of a target-derived neuronal cholinergic differentiation factor. Neuron 5:899-910.

Rao MS, Patterson PH, Landis SC (1992) Multiple cholinergic differentiation factors are present in footpad extracts: comparison with known cholinergic factors. Development 116:731-744.

Rao MS, Escary J, Sun Y, Perreau J, Patterson PH, Zigmond RE, Brulet P, Landis SC (1993) Leukemia inhibitory factor mediates an injury response but not a target-mediated developmental transmitter switch in sympathetic neurons. Neuron 11:1175-1185.

Roghani A, Shirzadi A, Butcher LL, Edwards RH (1998) Distribution of the vesicular transporter for acetylcholine in the rat central nervous system. Neuroscience 82:1195-1212.

Romano SJ, Corriveau RA, Schwarz RI, Berg DK (1997) Expression of the nicotinic receptor alpha 7 gene in tendon and periosteum during early development. J Neurochem 68:640-648.

Saadat S, Sendtner M, Rohrer H (1989) Ciliary neurotrophic factor induces cholinergic differentiation of rat sympathetic neurons in culture. J Cell Biol 108:1807-1816.

Sandhu HS, Herskovits MS, Singh IJ (1987) Effect of surgical sympathectomy on bone remodeling at rat incisor and molar root sockets. Anat Rec 219:32-38.

Sandhu HS, Kwong-Hing A, Herskovits MS, Singh IJ (1990) The effects of surgical sympathectomy on bone resorption in the rat incisor socket. Arch Oral Biol 35:1003-1007.

Schafer MK, Eiden LE, Weihe E (1998) Cholinergic neurons and terminal fields revealed by immunohistochemistry for the vesicular acetylcholine transporter. II. The peripheral nervous system. Neuroscience 84:361-376.

Schotzinger R, Landis SC (1988) Cholinergic phenotype developed by noradrenergic sympathetic neurons after innervation of a novel cholinergic target in vivo. Nature 335:637-639.

Schotzinger RJ, Landis SC (1990) Postnatal development of autonomic and sensory innervation of thoracic hairy skin in the rat. Cell Tissue Res 260:575-587.
Schotzinger RJ, Yin X, Landis SC (1994) Target determination of neurotransmitter phenotype in sympathetic neurons. J Neurobiol 25:620-639.

Singh IJ, Herskovits MS, Chiego DJ, Klein RM (1982) Modulation of osteoblastic activity by sensory and autonomic innervation of bone. In: Factors and mechanisms influencing bone growth (Dixon J, Sarnat M, eds), pp 535-551. New York: Liss.

Sjoqvist F (1963) The correlation between the occurrence and localization of acetylcholinesterase-rich cell bodies in the stellate ganglion and the outflow of cholinergic sweat secretory fibers to the fore paw of the cat. Acta Physiol Scand 57:339-351.

Specht LA, Pickel VM, Joh TH, Reis DJ (1981) Light-microscopic immunocytochemical localization of tyrosine hydroxylase in prenatal rat brain. I. Early ontogeny. J Comp Neurol 199:233-253.

Stevens LM, Landis SC (1987) Development and properties of the secretory response in rat sweat glands: relationship to the induction of cholinergic function in sweat gland innervation. Dev. Biol. 123:179-190.

Teitelman G, Baker H, Joh TH, Reis DJ (1979) Appearance of catecholamine synthesizing enzymes during development of the rat nervous system: possible role of tissue environment. Proc Natl Acad Sci USA 76:509-513.

Tyrrell S, Landis SC (1994) NPY and VIP expression in sympathetic neuroblasts and subsequent regulation of neuropeptide expression. J Neurosci 14:4529-4547.

Yamagata M, Sanes JR (1995) Target-independent diversification and target-specific projection of chemically defined retinal ganglion cell subsets. Development 121:3763-3776.

Yamamori T, Fukada K, Aebersold R, Korsching S, Fann MJ, Patterson PH (1989) The cholinergic neuronal differentiation factor from heart cells is identical to leukemia inhibitory factor. Science 246:1412-1416.

Yodlowski ML, Fredieu JR, Landis SC (1984) Neonatal 6-hydroxydopamine treatment eliminates cholinergic sympathetic innervation and induces sensory sprouting in rat sweat glands. J Neurosci 4:1535-1548. 